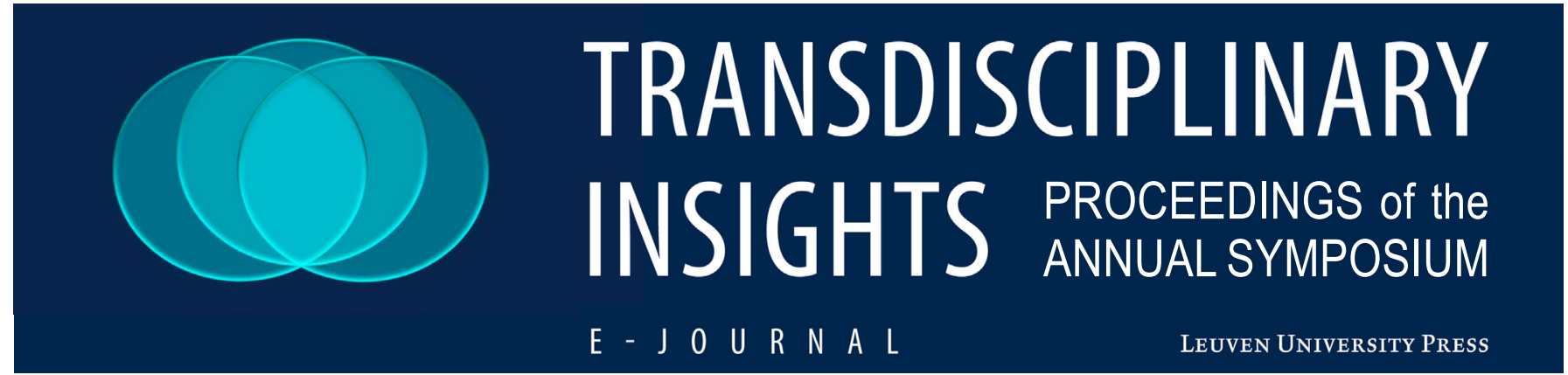

2021 KU Leuven Facing the Future: "How to increase societal impact?" https://rega.kuleuven.be/cev/Symposium/facing-the-future/Program-2021 Leuven, 5 May 2021
Program

17h15 - 17h20 Introduction by the Institute for the Future

17h20 - 17h45 STEAM+: Transforming higher education through collaborative play

17h45 - 18h10 InclusiVaart. (Re)defining shared neighborhood spaces $18 \mathrm{~h} 10-18 \mathrm{~h} 35$ Conceptualising Open Science in the 21st Century $18 \mathrm{~h} 35$ - $19 \mathrm{~h} 00$ Coronavirus Pandemic Preparedness

\title{
Conceptualising Open Science in the 21st Century
}

\section{Mercy Gloria Ashepet, ${ }^{1,2,3}$ Ignace Bossuyt, ${ }^{1,4}$ Hein Brookhuis, ${ }^{1,5}$ Cristian Constantin, ${ }^{1,6}$ Marta Fedele,,$^{1,7}$ Naina Goel, ${ }^{1,8,9}$ Daniel Pizzolato, ${ }^{1,10}$ Priscilla Van Even, ${ }^{1,11}$ Ana Barbosa Mendes, ${ }^{1,12^{\star}}$ Anne Snick ${ }^{1,13}$}

${ }^{1} \mathrm{KU}$ Leuven, Honours Programme Transdisciplinary Insights, Institute for the Future, Leuven, Belgium; ${ }^{2}$ Department of Biology, Royal Museum for Central Africa, Belgium;

${ }^{3}$ Department of Earth and Environmental science, Division of Bioeconomics, KU Leuven, Belgium;

${ }^{4}$ Department of Computer Science, NUMA, KU Leuven, Belgium;

${ }^{5}$ Cultural History Research Group, KU Leuven, Belgium; ${ }^{6}$ Department of Management, Strategy and Innovation, KU Leuven, Belgium;

${ }^{7}$ Faculty of Psychology and Educational Sciences, KU Leuven, Belgium;

${ }^{8} \mathrm{KU}$ Leuven Kulak, Laboratory of Aquatic Biology, Kortrijk, Belgium;

'University of Ghent, Faculty of Bioscience Engineering, Department of Animal Sciences and Aquatic Ecology, Ostend, Belgium;

\author{
${ }^{10}$ Centre for Biomedical Ethics and Law, Department of \\ Public Health and Primary Care, KU Leuven, Belgium; \\ ${ }^{11}$ Meaningful Interactions Lab (Mintlab), KU Leuven, \\ Belgium; \\ ${ }^{12}$ ITEC, an imec research group, Faculty of Psychology \\ and Educational Sciences, KU Leuven, Belgium; \\ ${ }^{13}$ Club of Rome - EU;
}

"Corresponding author: ana.barbosamendes@kuleuven.be.

Abstract

Open Science that is adaptive to the complexity of the 21 st century is emerging in transdisciplinary institutions outside of academia. Despite its growing popularity and plurality as a movement, the scope in which Open Science is practiced and discussed inside academia is still mostly restricted to the scientific community and fragmented between disciplinary silos. Researchers and policymakers promoting Open Science often focus on knowledge translation and still recognise experts and academia as the main producers of knowledge, essentially closing the research process to non-researchers 
and preventing other perspectives from being integrated into knowledge production. Our aim with this project was to adopt a systems perspective to understand how Open Science can address the challenges in the current knowledge production system. Open discussions among the team members revealed distinct understandings of what constitutes Open Science. Thus, during our process we collated these many definitions of Open Science and extracted the dimensions that underlie such definitions and mapped how these dimensions could be interconnected in a more comprehensive conceptualisation of Open Science. Future iterations of the challenge could build on our reflections and explore how these Open Science dimensions translate into scientific practice and how researchers can be encouraged to reflect on Open Science in a more systems-oriented way. Our findings have been summarised in a small video. ${ }^{1}$

\section{Key words}

reproducibility, systems approach, knowledge production system, sustainable research model, research wast

\section{Title of the original challenge}

Open Science and Reproducibility (https://rega.kuleuven.be/if/open-science-andreproducibility) 